\title{
Impact of the Position of Ectopic Ventricular Activation Origin on Its Noninvasive Assessment: A Simulation Study
}

\author{
Elena Deutsch ${ }^{1, *}$, Milan Tyšler ${ }^{1}$ and Peter Kneppo ${ }^{1}$ \\ 'Department of Biomedical Technology, Faculty of Biomedical Engineering, Czech Technical University in Prague, nám. Sítná 3105, 272 01 Kladno, \\ Czech Republic
}

\begin{abstract}
The accuracy of a method for noninvasive localization of the origin of premature ventricular complex (PVC) was tested on simulated data. The method is based on inverse solution in terms of a single dipole that uses integrals of body surface potentials (BSPs) and a model of the torso volume conductor. ECGSIM software was used to simulate ectopic activation in a realistic ventricular model and BSPs on an inhomogeneous torso. Ectopic activation origins were simulated in 120 positions located in anterior, lateral, posterior, and septal segments of the right and left ventricle (RV, LV). In both septal segments 15 endocardial positions were specified, in all other segments 5 epicardial, 5 endocardial, and 5 in the valvular region were defined. Corresponding BSPs for initial $5 \mathrm{~ms}$ interval of the ectopic activity were simulated in 300 torso nodes as well as in 192 points representing BSP mapping leads. Simulated BSPs and the torso model with lungs and heart cavities were then used for the inverse localization of each PVC origin. The localization error (LE) was defined as the distance between the simulated and inversely estimated PVC origin position. All simulated PVC origins were localized in the correct ventricular segment. There was no significant difference between the LEs in the RV and LV, except the septal segments where the LE was significantly smaller in the RV $(p<0.05)$. There were no significant differences between the LEs obtained using BSP maps from 300 torso nodes or 192 mapping leads (mean values $L E_{300}=5.2 \pm 4.4 \mathrm{~mm}$ and $L E_{192}=5.3 \pm 4.5 \mathrm{~mm}$ ).
\end{abstract}

Keywords: forward and inverse problem of electrocardiography; double layer model; dipole model; ECGSIM; ectopic ventricular activity; body surface potential mapping; noninvasive localization.

\section{Introduction}

Premature ventricular complexes (PVCs) or ventricular extrasystoles are manifestations of ectopic ventricular activity caused by some precocious impulse in the ventricular cells and resulting in irregular mechanical heart contractions impairing its pumping function. PVCs are generally induced by physical activity, hormonal changes in female patients, viral infection of the myocardium, drug effect, etc. [1, 2]. Accidental PVCs occur in many people, are mostly asymptomatic and do not pose any danger in healthy individuals. At the same time, frequent PVCs, defined as more than $20 \%$ of all QRS complexes, can lead to a form of tachycardia related cardiomyopathy, called PVC-related cardiomyopathy [3]. Since occurrence of PVCs increases in general adult population with aging and they are common in patients with cardiomyopathy, hypertension, ventricular hypertrophy and mitral valve prolapse, PVCs present a risk of fatal rhythm disturbances [4]. 
A conventional approach to reduce the PVCs occurrence is pharmacological therapy that modifies the action potential shape, the conductivity and the refractory period of cardiac cells. In cases when antiarrhythmic drugs do not succeed, a radiofrequency ablation (RFA) is performed [5]. RFA is a procedure for destruction of cardiac cells by applying $300-1000 \mathrm{kHz}$ radiofrequency current that heats the tissue and creates a hyperthermic lesion [6]. The current is applied via an intracardiac catheter that is guided under $\mathrm{X}$-ray control and optionally by a 3D navigational system in the catheterization laboratory. In order to find the origin of the PVC, activation mapping and pace mapping techniques are used. The first one identifies the location with the earliest electrical activation and the second one compares the morphology of a paced QRS complex with the morphology of the PVC in all ECG leads [7]. This approach to finding the PVC origin can be time consuming, putting the patient and medical stuff under additional $X$-ray exposure.

We are developing a method that uses multichannel ECG measurement and information about the torso structure for noninvasive localization of the PVC origin already before the RFA. In this way it would be possible to decrease the time of the invasive procedure with $\mathrm{X}$-ray exposure and minimize the risks for the patient $[8,9]$. In order to find the PVC origin, this method solves the socalled inverse problem of electrocardiography. Mathematically, the inverse solution describes the electrical activity of the heart using a model that represents the heart as an electrical generator and the torso as a volume conducting medium [10]. The heart can be represented by a single dipole model, multiple dipoles, equivalent double layer, transmembrane voltages or epicardial potentials. Generally, the inverse problem is ill-posed, i.e. its solution might not be unique and/or might not depend continuously on the input data [11]. To solve this issue, regularization methods limiting the mathematical model are used [12-14]. In the case of PVC origin, a small activated region is localized and the cardiac generator can be modeled by a single dipole [15].

The aims of this simulation study were (1) to estimate the best achievable accuracy of the method when high enough number of measured surface points is available and the measurement errors in the input potentials and geometry data are negligible and (2) to check how the accuracy of the localization depends on the real PVC origin position in the ventricles.

\section{Methods and Materials}

\subsection{Simulation of the Ectopic Ventricular Activation}

Realistically shaped ventricular model and inhomogeneous torso model with lungs and heart cavities created from a patient MR scan (Figure 1a) were used to simulate the PVCs. Relative conductivities of the torso, lungs, and heart cavities were 1.0, 0.2, and 3.0, respectively [16]. One hundred and twenty individual PVC origins were defined in eight segments of the right and left ventricle (RV, LV): anterior RV (RV Ant), lateral RV (RV Lat), posterior RV (RV Post), septal RV (RV Sept), anterior LV (LV Ant), lateral LV (LV Lat), posterior LV (LV Post), and septal LV (LV Sept) (Figure 2). In septal segments fifteen endocardial positions of the PVC origins were simulated, in all other segments 5 epicardial (epi), 5 endocardial (endo), and 5 positions in the valvular region were specified.

Freely available software ECGSIM [17] was used to simulate the ventricular activation sequence and corresponding body surface potentials (BSPs). The software allows modification of the heart generator model by adjusting local action potentials (durations and amplitudes), depolarization and repolarization times, or transmembrane potentials. Changes are projected on the heart surface and in BSPs. ECGSIM solves the forward problem of electrocardiography using an equivalent double layer model of the heart generator, and the boundary element method for computation of BSPs using the transfer matrix $A_{i j}$ containing transfer coefficients describing the surface potential $\phi_{j}(t)$ in point $j$ and time instant $t$, generated by an elementary current double layer source at a ventricular node $i$, pointing towards the ventricular mass [18]:

$$
\phi_{j}(t)=\sum_{i=1}^{N} \mathbf{A}_{i j} S_{i}(t)
$$

where $S_{i}(t)$ is the source strength in $i$-th node of the myocardial surface in time instant $t$.

The BSPs were calculated in all 300 nodes of the torso model as well as in 192 torso points representing positions of a BSP mapping lead 
system. The torso model had higher density of the nodes in the near heart region (Figure 1b) where is the area of the highest electrical gradient. The BSP mapping lead system was formed by 16 vertical stripes of 12 electrodes placed regularly around the torso (Figure 1c) with the intention to have maximum coverage of the torso and still be practical enough to be used in clinical practice. Integral BSP maps (IBSPM) were obtained for both, torso model nodes or BSP mapping leads positions from the potentials in all nodes or leads (Figure $1 b, c)$ :

$$
I B S P M=\int_{I} \phi_{j}(t) d t
$$

where $I$ - is the examined time interval.

\subsection{Noninvasive Localization of the PVC Origin}

The main goal of the proposed and tested method for noninvasive localization of the initial pathological ventricular activation by the inverse solution is to provide the physician accurate diagnostic information about the location of the PVC origin before the RFA. It is supposed, that the initial activation occurs in small cardiac region which can be represented by a single dipole [19]. To avoid solving nonlinear problem of finding the dipole moments and position and to speed up the inverse solution, the dipole moments are searched in predefined possible positions in a regular rectangular $3 \mathrm{~mm}$ grid within the ventricular myocardium volume (Figure 3c). The input data for the inverse solution are integral BSP map and information about the inhomogeneous torso model (torso, lungs, and ventricular cavities with relative conductivities of $1.0,0.25$ and 3.0, respectively). Then, the inverse solution for a single dipole in a predefined position in the ventricles can be calculated using the equation:

$$
G^{\prime}=\mathbf{B}^{+} I B S P M
$$

where $G^{\prime}$ - is the dipolar equivalent generator, $\mathbf{B}^{+}$ - is the pseudo-inverse of the transfer matrix $\mathbf{B}$ representing the relation between the equivalent heart generator and potentials on the torso, and IBSPM is integral BSP map from the initial $5 \mathrm{~ms}$ interval of the ectopic ventricular activation (Figure 3a).

The same inhomogeneous torso model with lungs and heart cavities (having the relative conductivities of 0.25 for the lungs and 3.0 for the blood in ventricular cavities) was used as in the forward simulations. As the main goal of this study was to estimate the best achievable localization accuracy of the PVC origin, no additional noise was added to the simulated BSPs and no geometrical fluctuations of the torso model were introduced. The evaluated time interval $I$ representing the initial $5 \mathrm{~ms}$ of the ectopic activation was short enough to guarantee that the activated ventricular area is still so small that it can be represented by a dipole.

For each PVC origin, the inverse problem was solved for all predefined positions of the single dipole. The solution was selected as the best position of the equivalent dipole based on the criterion of minimal relative residual error (RRE) between the input IBSPM and integral BSP map computed from the inversely obtained dipole generator $G^{\prime}(G m)$, which can be calculated using the equation:

$$
R R E=\sqrt{\sum_{i=1}^{n}\left(I B S P M_{i}-G m_{i}\right)^{2}} / \sqrt{\sum_{i=1}^{n} I B S P M_{i}^{2}}
$$

where $n$ is the number of points in the IBSPM.

\subsection{Accuracy of the Inverse Solution}

The accuracy of the inverse solution was evaluated using a localization error (LE) defined as the distance between the simulated PVC origin location and the inversely obtained location of the equivalent dipole generator. Because the positions of the inversely obtained dipoles were in a discrete grid and the locations of the simulated PVC origins could be located arbitrarily on the ventricular surface, the error of the model (ME) was also defined as the distance between the simulated PVC origin location and location of the nearest possible dipole position in the grid.

\section{Critical Success Factors}

Fifteen positions of the PVC origins were simulated in each of the eight segments of both heart ventricles, resulting in 120 PVC origins in total. The forward problem was solved by the ECGSIM software using the equivalent double layer model. The inverse problem was solved using equivalent single dipole model of the heart generator, inhomogeneous torso model and integral BSP 


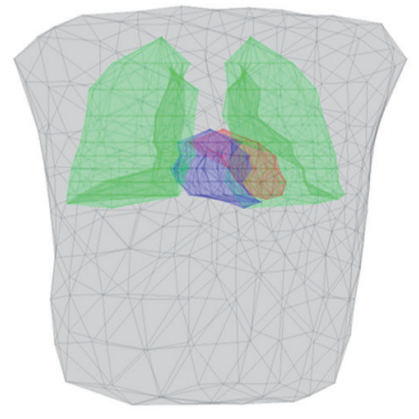

a

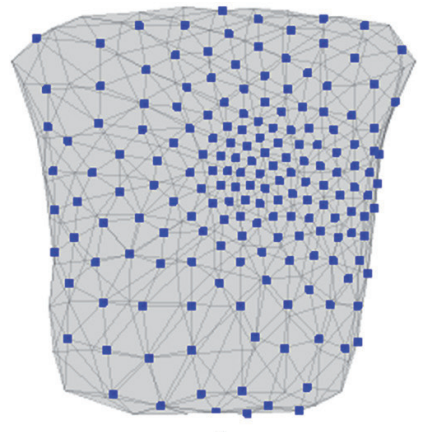

b

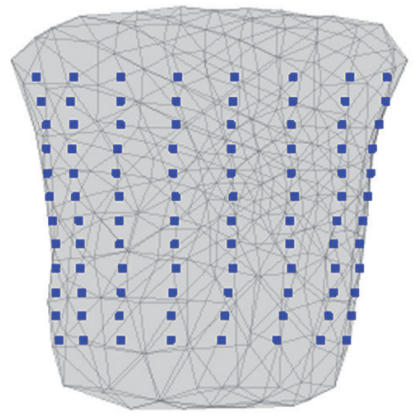

C

Fig. 1: (a) Inhomogeneous torso model including lungs and right and left ventricle. Anterior view. (b) Torso model with marked positions of the nodes. Anterior view. (c) Torso model with positions of the 192 BSP mapping leads (12 electrodes in 16 stripes). Anterior view.

$$
\begin{array}{cc}
\square \text { - RV Ant } & * \text { - LV Ant } \\
\star \text { - RV Lat } & \diamond \text { - LV Lat } \\
\Delta \text { - RV Post } & \nabla \text { - LV Post } \\
\text { O-RV Sept } & * \text { - LV Sept }
\end{array}
$$

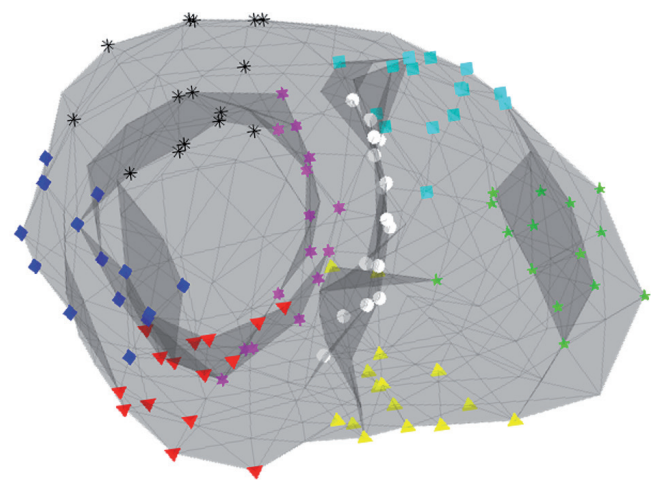

Fig. 2: Model of the heart ventricles with 120 simulated positions of PVC origins in eight selected ventricular segments. Base to apex view.
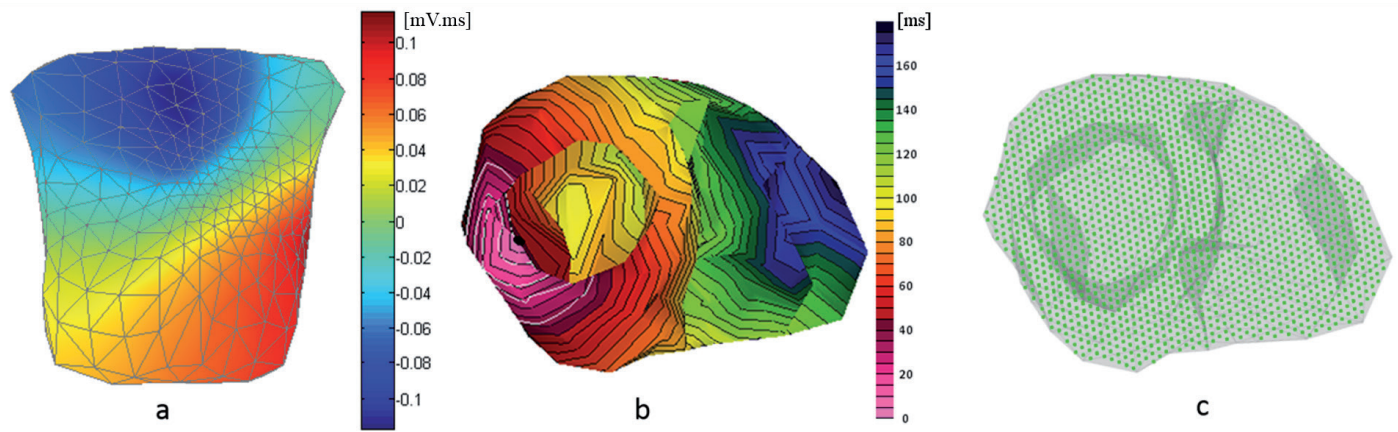

Fig. 3: (a) Torso model with integral body surface potential map computed from the initial 5 ms time interval for the simulated PVC origin in LV Lat2 location. (b) Model of the heart ventricles with isochrone map of the simulated activation started in LV Lat2 location. Base to apex view. (c) Model of the heart ventricles with predefined possible positions of the dipoles in a $3 \mathrm{~mm}$ grid used for the inverse solution. Base to apex view.

map IBSPM as input data. The IBSPM was computed for initial $5 \mathrm{~ms}$ time interval to represent the initial ectopic activity. Distance between the simulated PVC origin location and its inverse localization was assessed as the LE. Fisher's test was used to compare dispersions of LEs statistically.

The LE varied from $0.36 \mathrm{~mm}$ to $31.95 \mathrm{~mm}$ when the inverse solution was obtained using IBSPMs from 300 torso nodes and from $0.60 \mathrm{~mm}$ to 31.95 mm when 192 BSP mapping leads were used. The results of the inverse solution obtained when using different lead systems varied in 20 cases out of the 120 simulations (16\%), but the differences were not statistically significant. Only in 4 from 


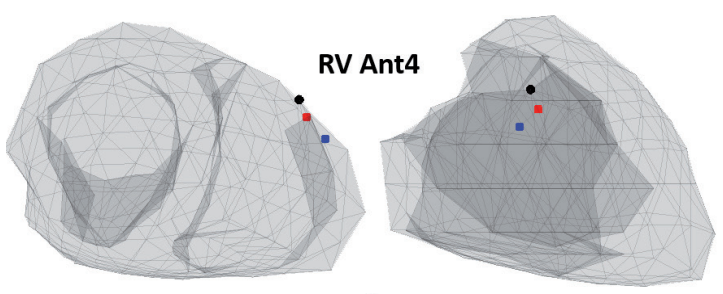

a

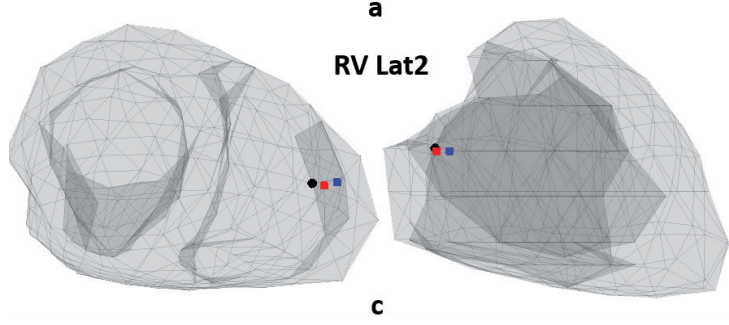

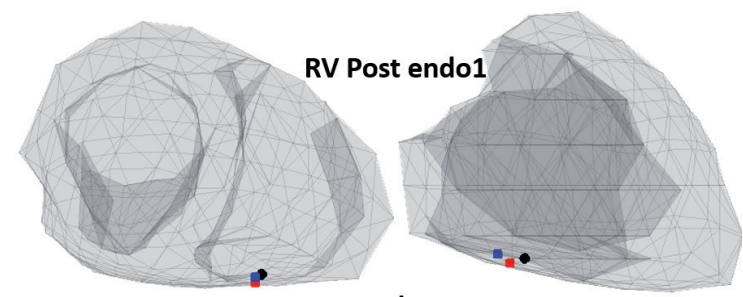

b

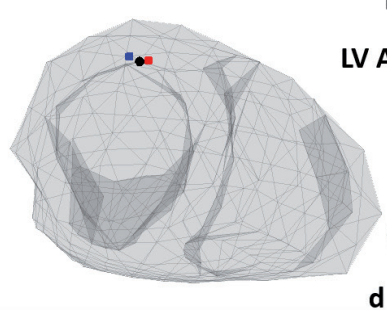

Ant1

Fig. 4: Four cases of the simulated PVC origin in which the difference between the inverse solutions obtained from 300 nodes and from 192 leads was higher than the $3 \mathrm{~mm}$ resolution of the method. Simulated PVC origin is marked by the black dot, its inversely obtained positions when using 300 nodes and 192 leads are marked by red and blue squares, respectively. (a) Case of PVC origin in the anterior segment of the RV (base to apex and right anterior oblique view). (b) Case of PVC origin at the endocardium of the posterior segment of the RV (base to apex and right anterior oblique view). (c) Case of PVC origin in the lateral segment of the RV (base to apex and right anterior oblique view). (d) Case of PVC origin in the anterior segment of the LV (base to apex and left anterior oblique view).
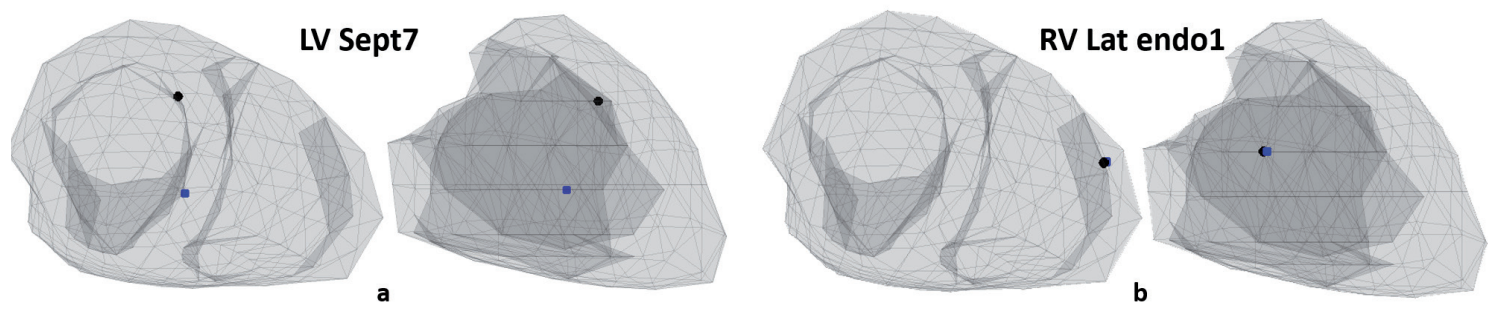

Fig. 5: Two cases of the simulated PVC origins with highest and smallest LE. Simulated PVC origins are marked by the black dot; its inversely obtained positions when using 300 nodes and 192 leads were in the same position and are marked by blue squares. (a) Case of PVC origin in the septal segment of the LV with highest LE (base to apex and right anterior oblique view). (b) Example of the PVC origin with smallest $L E$ at the endocardium of the lateral segment of the RV (base to apex view and right anterior oblique view).

the above mentioned 20 cases, the distance between the results obtained using different lead systems was higher than the $3 \mathrm{~mm}$ resolution of the method (Figure 4). In 40 cases when using 300 nodes and 38 cases when using 192 leads, the LE was identical with the ME, i.e. the inverse method was able to find the best possible solution at given $3 \mathrm{~mm}$ method resolution.

The highest LE (Figure 5a) was observed for the PVC origin in the septum of the LV (LELV Sept7= $31.95 \mathrm{~mm}$ ). The smallest LE (equal to the ME) for both used sets of BSPs was observed in 38 cases. An example is given in Figure $5 b$ for the PVC origin in the lateral segment of the RV (LERV Lat endol= $1.32 \mathrm{~mm}$ ).

There was also no significant difference between
LEs of PVC origins in the RV and LV for both sets of BSPs (300 nodes and 192 leads). The mean LE of PVC origins in the RV was smaller when signals from 300 nodes were used (RV: LE $300=4.69 \pm 3.95 \mathrm{~mm}$ ) compared with mean LE when 192 leads were used (RV: $L E_{192}=5.07 \pm 4.24 \mathrm{~mm}$ ) but the difference was not significant. The mean LE of PVC origins in the LV was $5.61 \pm 4.85 \mathrm{~mm}$ when 300 nodes were used and $5.62 \pm 4.67$ when 192 leads were used (Figure 6).

The obtained results are shown in more detail in Figure 7 where the means and standard deviations of the LEs obtained for PVC origins in different regions (epicardial, endocardial and valvular region) of four ventricular segments are presented. As it can be seen, the LE values for different locations were very similar when the 300 torso nodes or 192 
leads were used as input data. The lowest values were obtained for PVC origins with endocardial position in all segments except the RV Lateral. The LE of origins in the RV Septal was lower than in the LV Septal for both BSP sets $(p<0.05)$.

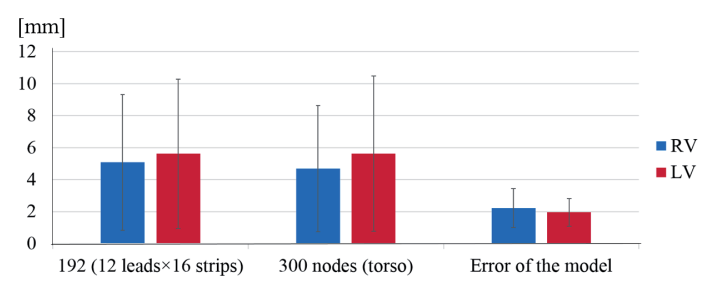

Fig. 6: Mean and standard deviation of the LE of PVC origins in the $R V$ and $L V$

Even thou the simulation study of the PVC origins localization using the proposed inverse solution in most cases did not show significant difference between the LEs obtained for different regions, it has to be kept in mind that the simulation study was performed in a model with no noise in the signals. In the clinical practice, where the noise is unavoidable part of the measurement, the selected time interval representing ectopic activity should be of longer duration. Nevertheless, our study in patients [15] had shown that using this method and increasing the time interval for IBSPM computation up to $15 \mathrm{~ms}$ allows to localize the PVC origin with a LE within $2 \mathrm{~cm}$. As the obtained mean LE using noise-free input data was about 5-6 $\mathrm{mm}$, the study also suggests that smaller grid dimension for the possible positions of the PVC origin would probably not improve the accuracy of the method.

\section{Conclusions}

The simulation study showed that if the disturbances in the input data can be neglected, the proposed method for noninvasive localization of the PVC origin based on inverse solution in terms of a single dipole is robust enough and all PVC origins were located in correct ventricular segments. The average LE of the localization was 5 to $6 \mathrm{~mm}$, nearly the same for the RV and LV. For PVC origins in the septal segments the LE was lower in the RV than in the LV. Generally, the simulations showed that the LEs are higher for PVC origins in heart regions that are located more distantly from the body surface but this difference was statistically not significant. There was also no significant difference between the LEs obtained for solutions using all 300 torso nodes or 192 BSP mapping leads what justifies the application of the method on practically measurable data.

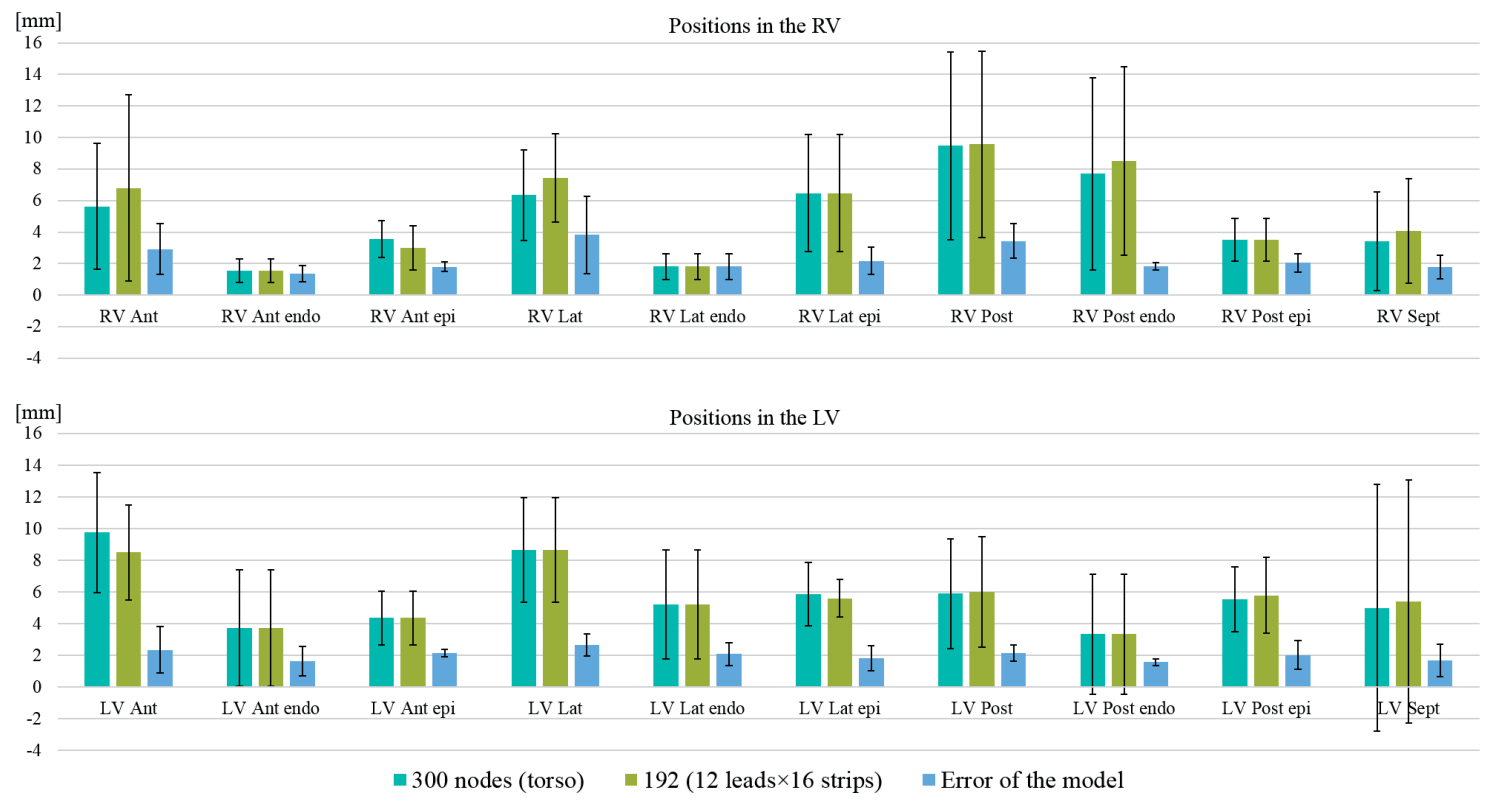

Fig. 7: Mean and standard deviation of the LE and ME for PVC origins located in the RV (top) and LV (bottom) in different regions (epicardial, endocardial, valvular) in four ventricular segments: anterior, lateral, posterior, septal. 


\section{Acknowledgments}

The present study was supported by research grants 17110/122/1222100G000 from the Department of Biomedical Technology Faculty of Biomedical Engineering, CTU in Prague, Czech Republic, APVV-14-0875 from the Research and Development Agency, and 2/0071/16 from the VEGA Grant Agency in Slovakia.

\section{References and Notes}

[1] Adams, J.C., Srivathsan, K., Shen, W.K. (2012). Advances in management of premature ventricular contractions. Journal of interventional cardiac electrophysiology: an international journal of arrhythmias and pacing, 35, 2, 137-149.

[2] Hsing, J., Wang, P.J., Al-Ahmad, A. (2009). Electroanatomical mapping. Al-Ahmad, A., Callans, D.J., Hsia, H., Natale, A. Electroanatomical mapping technologies. Blackwell Publishing, Singapore.

[3] Ban, J.-E., Park, H.-C., Park, J.-S., Nagamoto, Y., Choi, J.-I., Lim, H.-E., Park, S.-W., Kim, Y.-H. (2013). Electrocardiographic and electrophysiological characteristics of premature ventricular complexes associated with left ventricular dysfunction in patients without structural heart disease. Europace, 15, 5, 735-741.

[4] Clifford, G.D., Azuaje, F., McSharry, P.E. (2006). Advanced methods and tools for ECG data analysis. Artech House, Norwood, MA, USA.

[5] Fogoros, R.N. (2006). Electrophysiologic testing. Blackwell Publishing, Malden, Mass.

[6] Antzelevitch, C., Burashnikov, A. (2011) Overview of basic mechanism of cardiac arrhythmia. Cardiac Electrophysiology Clinics, 3, 1, 23-45.

[7] Huang, S.K., Wood, M.A. (2006). Catheter ablation of cardiac arrhythmias. Saunders/Elsevier, Philadelphia.

[8] Farina, D., Dossel, O. (2009). Non-invasive model-based localization of ventricular ectopic centers from multichannel ECG. International Journal of Applied Electromagnetics and Mechanics, 30, 3-4, 289-297.

[9] Lai, D., Sun, J., Li, Y., He, B. (2013). Usefulness of ventricular endocardial electric reconstruction from body surface potential maps to noninvasively localize ventricular ectopic activity in patients. Physics in Medicine and Biology, 58, 11, 3897-3909.

[10] Malmivuo, J., Plonsey, R. (1995). Bioelectromagnetism. Oxford University Press, New York, New York.

[11] Gulrajani, R.M. (1998). The forward and inverse problems of electrocardiography. IEEE Engineering in Medicine and Biology, 17, 5, 84-122.

[12] Brooks, D.H., Ahmad, G.F., MacLeod, R.S., Maratos, G.M. (1999). Inverse electrocardiography by simultaneous imposition of multiple constraints. IEEE Transactions on Biomedi- cal Engineering, 46, 1, 3-18.

[13] Potyagaylo, D., Cortes, E.G, Schulze, W.H.W., Dossel, O. (2014). Binary optimization for source localization in the inverse problem of ECG. Medical \& Biological Engineering \& Computing, 52, 9, 717-728.

[14] Shou, G., Xia, L., Liu, F., Jiang, M., Crozier, S. (2011). On epicardial potential reconstruction using regularization schemes with the L1-norm data term. Physics in medicine and biology, 56, 1, 57-72.

[15] Punshchykova, O., Svehlikova, J., Tysler, M., Grunes, R., Sedova, K., Osmancik, P., Zd’arska, J., Herrman, D., Kneppo, P. (2016). Influence of torso model complexity on the noninvasive localization of ectopic ventricular activity. Measurement Science Review, 16, 2, 96-102.

[16] Oostendorp T.F., van Oosterom A. (2004) ECGSIM: an Interactive Tool for the Study of the Relation between the Electric Activity of the Heart and the QRST Waveforms at the Body Surface. The 26th Annual International Conference of the IEEE Engineering in Medicine and Biology Society, 3559-3562.

[17] PEACS. ECGSIM software, from http://www.ecgsim.org, 15.02.2018.

[18] Huiskamp, G., van Oosterom, A. (1988) The Depolarization Sequence of the Human-Heart Surface Computed from Measured Body-Surface Potentials. IEEE Transactions on Biomedical Engineering, 35, 12, 1047-1058.

[19] Tysler M., Tinova M. (1993) Representation of myocardium depolarization by simple models. Computers in cardiology, 703-706.

\section{Biographical notes}

Elena Deutsch, MSc., (1988) is currently an assistant and a PhD. student in Biomedical and Clinical Technology at the Czech Technical University in Prague, Faculty of Biomedical Engineering, Department of Biomedical Technology. In 2010 she graduated with MSc. degree in Medical Devices and Systems from the National Technical University of Ukraine "Igor Sikorsky Kyiv Polytechnic Institute". Topic of her dissertation thesis is noninvasive localization of ectopic ventricular activity. She was a lecturer in NTUU "KPI" in 2011 and an engineer in Laboratory of Cardiology and Electrophysiology of the state establishment "National M. Amosov Institute of Cardiovascular Surgery of the Academy of Medical Sciences of Ukraine" from 2007 till 2011. From 2015 she works as a researcher focusing on body surface potential mapping and noninvasive localization of premature ventricular complexes.

Assoc. prof. Milan Tyšler, MSc., PhD., (1951) is director of the Institute of Measurement Science, Slovak Academy of Sciences in Bratislava. He obtained MSc. in Electrical Engineering from the Faculty of Electrical Engineering, Slovak University of Technology in Bratislava in 1974, PhD. in Measurement Science from the Institute of Measurement Theory, Slovak Academy of Sciences in 1982 and became the associate professor of the Faculty of Mechanical Engineering, Technical University in Košice in 2006. He is member of scientific boards of the 
Acta Mechanica Slovaca

Journal published by Faculty of Mechanical Engineering - Technical University of Košice

FEE SUT in Bratislava and Faculty of Biomedical Engineering, Zzech Technical University in Prague, Kladno. His professional interests include modeling of the cardiovascular system and development of methods and devices for biosignal measurement and processing. He is secretary and Council member of the International Society of Electrocardiology, member of the IEEE EMBS Society, president of the Slovak Society of BME and Medical Informatics and Slovak IMEKO Committee. He was president of five international conferences Measurement and International Congress on Electrocardiology. He is member of the Measurement Science Review editorial board.

Prof. Peter Kneppo, MSc., DrSc., (1940) is head of Department of Biomedical Technology at Faculty of Biomedical Engineering, (zech Technical University in Prague. He obtained MSc. in Automatics and Computer Engineering from Institute of Automatics and Computer Engineering, Moscow Power Engineering Institute in 1964, PhD. degree in Measurement Science from the Institute of Measurement Science, Slovak Academy of Sciences in 1972, DrSc. from Slovak Academy of Sciences in 1983, assoc. prof. from Faculty of Mechanical Engineering, Technical University in Košice in 1996 and prof. from Faculty of Mechanical Engineering, Technical University in Košice in 2001. He is member of the scientific board of Faculty of Biomedical Engineering, CTU in Praque. His professional interests include electric and magnetic measurement and mapping of heart activity, system modeling, data acquisition and processing, automation and regulation. He is a senior member of the IEEE Society, member of the IMEKO Committee, delegate of the IFMBE General Assembly, member of the Polimetrica advisory board (Italy) and Elsevier Measurement editorial board. 